\title{
Three-wave coupling coefficients for a magnetized plasma
}

\author{
$\underline{\text { G. Brodin }}^{1}$ and L. Stenflo ${ }^{2}$ \\ ${ }^{1}$ Department of Physics, Umeå University, SE-901 87 Umeå, Sweden \\ ${ }^{2}$ Department of Physics, Linköping University, SE-581 83 Linköping, Sweden
}

\begin{abstract}
The resonant interaction between three waves in a uniform magnetized plasma is reconsidered. Starting from previous kinetic expressions, that contain a general but too little used result, we are able to improve the formulas. This leads to an explicit expression for the three wave coupling coefficient which applies for arbitrary wave propagation in a magnetized Vlasov plasma.
\end{abstract}

\section{Introduction}

The resonant interaction between three waves in a plasma has now been studied during more than half a century. The first review of such processes in an unmagnetized plasma [1] was followed by specific studies for a magnetized plasma. In Ref. [2], a general theory for wave interactions in a cold one-component plasma was then developed. The numerous subsequent improvements of the theory were later reviewed in Refs. [3] and [4].

However, partly due to the lack of simple explicit expressions for the coupling coefficients, the production of alternative formulas continued. In the present paper we are going to discuss another expression which can be considered as the final result of previous efforts.

\section{Results}

Considering the resonant interaction between three waves with frequencies $\omega_{j}(j=1,2,3)$ and wave vectors $\mathbf{k}_{j}$, we assume that the matching conditions

$$
\omega_{3}=\omega_{1}+\omega_{2}
$$

and

$$
\mathbf{k}_{3}=\mathbf{k}_{1}+\mathbf{k}_{2}
$$

are satisfied. When calculating the coupling coefficients, it turns out that they contain a common factor V. It is then possible to write the three coupled equations as

$$
\frac{d W_{1,2}}{d t}=-2 \omega_{1,2} \operatorname{ImV}
$$

and

$$
\frac{d W_{3}}{d t}=2 \omega_{3} \operatorname{ImV}
$$

where $W=\varepsilon_{0} \mathbf{E}^{*} \cdot(1 / \omega) \partial\left(\omega^{2} \boldsymbol{\varepsilon}\right) \mathbf{E}$ is the wave energy, $\boldsymbol{\varepsilon}$ is the usual textbook dielectric tensor, and $\operatorname{ImV}$ stands for the imaginary part of $\mathrm{V}$ where ([3])

$$
\mathrm{V}=\sum_{s} m \int d \mathbf{v} \mathbf{F}_{0}(\mathbf{v}) \sum_{\substack{p_{1}+p_{2}=p_{3} \\ p_{j}=0, \pm 1, \pm 2, \ldots}} I_{1}^{p_{1}} I_{2}^{p_{2}} I_{3}^{-p_{3}}\left[\frac{\mathbf{k}_{1} \cdot \mathbf{u}_{1 p_{1}}}{\omega_{1 d}} \mathbf{u}_{2 p_{2}} \cdot \mathbf{u}_{3 p_{3}}^{*}+\frac{\mathbf{k}_{2} \cdot \mathbf{u}_{2 p_{2}}}{\omega_{2 d}} \mathbf{u}_{1 p_{1}} \cdot \mathbf{u}_{3 p_{3}}^{*}+\frac{\mathbf{k}_{3} \cdot \mathbf{u}_{3 p_{3}}^{*}}{\omega_{3 d}} \mathbf{u}_{1 p_{1}} \cdot \mathbf{u}_{2 p_{2}}\right.
$$




$$
\left.-\frac{i \omega_{c}}{\omega_{3 d}}\left(\frac{k_{2 z}}{\omega_{2 d}}-\frac{k_{1 z}}{\omega_{1 d}}\right) \mathbf{u}_{3 p_{3}}^{*} \cdot\left(\mathbf{u}_{1 p_{1}} \times \mathbf{u}_{2 p_{2}}\right)\right]
$$

where $\omega_{j d}=\omega_{j}-k_{j z} v_{z}-p_{j} \omega_{c}, I_{j}\left(=\exp \left(i \theta_{j}\right)\right)=\left(k_{j_{x}}+i k_{j y}\right) / k_{j \perp}$, and the velocity $\mathbf{u}_{j p_{j}}$ satisfies

$$
\begin{aligned}
\omega_{j d} \mathbf{u}_{j p_{j}}+i \omega_{c} \widehat{\mathbf{z}} \times \mathbf{u}_{j p_{j}}= & \frac{i q}{m \omega_{j}}\left\{\omega_{j d} J_{p_{j}} \mathbf{E}_{j}+\left[\left(v_{z} E_{j z}+\frac{p_{j} \omega_{c}}{k_{j \perp}^{2}} \mathbf{k}_{j \perp} \cdot \mathbf{E}_{j \perp}\right) J_{p_{j}}+\right.\right. \\
& \left.\left.\frac{i v_{\perp} \omega_{c}}{k_{j \perp}^{2}}\left(\widehat{\mathbf{z}} \times \mathbf{k}_{j}\right) \cdot \mathbf{E}_{j} \frac{d}{d v_{\perp}} J_{p_{j}}\right] \mathbf{k}_{j}\right\}
\end{aligned}
$$

where $J_{p_{j}}=J_{p_{j}}\left(k_{j \perp} v_{\perp} / \omega_{c}\right)$ denotes a Bessel function of order $p_{j}$.

The general theory for resonant three-wave interactions in plasmas then shows that the growth rate $\gamma$ can be determined from [3]

$$
\gamma^{2}=\frac{\omega_{1} \omega_{2}|\mathrm{~V}|^{2}}{W_{1} W_{2}}
$$

Eq. (7) is very useful when rather complex kinetic effects are involved, for example in the interaction between two kinetic Alfvén waves and one ion-sound wave [5].

The development of, for example, the z-components $\left(E_{j z}\right)$ of the wave electric field amplitudes is here governed by the three coupled bilinear equations (e.g. [3])

$$
\begin{aligned}
& \frac{d E_{1 z}^{*}}{d t}=\alpha_{1} E_{2 z} E_{3 z}^{*} \\
& \frac{d E_{2 z}^{*}}{d t}=\alpha_{2} E_{1 z} E_{3 z}^{*}
\end{aligned}
$$

and

$$
\frac{d E_{3 z}}{d t}=\alpha_{3} E_{2 z} E_{1 z}
$$

where the z-axis is along the external magnetic field $\left(B_{0} \widehat{\mathbf{z}}\right)$, the star denotes complex conjugate, $\alpha_{j}$ are the coupling coefficients, $d / d t=\partial / \partial t+\mathbf{v}_{g j} \cdot \nabla+v_{j}$ where $\mathbf{v}_{g j}$ is the group velocity of wave $j$, and $v_{j}$ accounts for the linear damping rate. Formulas that determine the coefficients $\alpha_{j}$ for a magnetized Vlasov plasma have been derived previously [3], although in rather inexplicit forms. It is the purpose of the present paper to point out that explicit expressions for $\alpha_{j}$ can also be deduced from those formulas.

Starting from Eqs. (12)-(14) of Ref. [3] it turns out that we can write $\alpha_{j}$ in the comparatively simple form

$$
\alpha_{1,2}=\frac{M_{1,2}}{\partial D\left(\omega_{1,2}, \mathbf{k}_{1,2}\right) / \partial \omega_{1,2}} C
$$

and

$$
\alpha_{3}=-\frac{M_{3}}{\partial D\left(\omega_{3}, \mathbf{k}_{3}\right) / \partial \omega_{3}} C
$$

where the general coupling constant $C$ as well as $D$ and $M_{j}$ are given by Ref. [6].

As a specific example we first mention the limiting case where all the waves are electrostatic. In that limit Eq. (6) reduces to 


$$
\mathbf{u}_{j p_{j}}=-\frac{i q}{m \omega_{j d} k_{z j}\left(1-\omega_{c}^{2} / \omega_{j d}^{2}\right)}\left(\mathbf{k}_{j}-\frac{i \omega_{c}}{\omega_{j d}} \widehat{\mathbf{z}} \times \mathbf{k}_{j}-\frac{\omega_{c}^{2}}{\omega_{j d}^{2}} k_{z j} \widehat{\mathbf{z}}\right) J_{p_{j}}
$$

whereas $M_{j}$ in (9) is

$$
M_{j}=\frac{k_{z j}^{2} k_{\perp j}^{2} c^{4}}{\omega_{j}^{2}}
$$

and the dispersion function $D\left(\omega_{j}, \mathbf{k}_{j}\right)$ is described by the wellknown formula (c.f. Ref. [3])

$$
D\left(\omega_{j}, \mathbf{k}_{j}\right)=\left(1+\sum_{s, p} \frac{q^{2}}{m \varepsilon_{0} k_{j}^{2}} \int \frac{d \mathbf{v}}{\omega_{j d}}\left(\frac{p \omega_{c}}{v_{\perp}} \frac{\partial F_{0}}{\partial v_{\perp}}+k_{z j} \frac{\partial F_{0}}{\partial v_{z}}\right) J_{p}^{2}\right) \frac{k_{j}^{4} c^{4}}{\omega_{j}^{4}}
$$

Thus the expressions for $\alpha_{j}$ are comparatively simple in the electrostatic limit.

Finally, we point out that Eqs. (10)-(12) have recently been generalized [6] so that arbitrary wave propagation is now also included. As the corresponding expressions for $\alpha_{j}$ are lengthy, we refer the reader to Ref. [6].

\section{Conclusions}

In the present paper we have improved the limiting results for three wave interactions in a cold plasma and pointed out that the explicit expressions for the coupling coefficients for wave interactions in a hot magnetized Vlasov plasma have been found recently. Our coupling coefficient $C$ can thus be used as a starting point to estimate the coupling strength where the interaction between any kind of waves in a plasma has to be considered. It can also be useful in interpretations of stimulated scattering of electromagnetic waves in space plasmas. In the latter case we refer the reader to a short historical account of stimulated electromagnetic emissions in the ionosphere [7]. Our results can also play a key role in the theoretical interpretations of laser-fusion experiments.

\section{References}

[1] Sagdeev, R.Z. and Galeev, A.A., Lectures on the non-linear theory of plasma, IC/66/64 (Int. Centre for Theor. Physics, Trieste, Italy 1964).

[2] Stenflo, L., 1973 Planet. Space Sci. 21, 391.

[3] Stenflo, L., 1994 Phys. Scr. T 50, 15.

[4] Shukla, P.K., 1999 Phys. Scr. T 82, 1.

[5] Chen, L. and Zonca, F., 2011 EPL 96, 35001.

[6] Brodin, G. and Stenflo L., 2012 Phys. Scr. 85, 035504.

[7] Stenflo, L., 2004 Phys. Scr. T 107, 262. 